\title{
Surveying social adversity in pregnancy: the antenatal risk burden experienced by
}

\section{Australian women}

Manuscript type: Original article

Prof Sharon Goldfeld

Murdoch Childrens Research Institute, the Centre for Community Child Health, The Royal Children's Hospital and The Department of Paediatrics, The University of Melbourne.

50 Flemington Road, Parkville, VIC 3052, Australia

Dr Elise D'Abaco

Melbourne Medical School, The University of Melbourne.

Grattan St, Melbourne VIC 3010.

Ms Hannah Bryson

Murdoch Childrens Research Institute, and the Centre for Community Child Health, The Royal Children's Hospital.

50 Flemington Road, Parkville, VIC 3052, Australia

Dr Fiona Mensah

Clinical Sciences and Biostatistics Unit, Murdoch Children's Research Institute, The Royal Children's Hospital.

50 Flemington Road, Parkville, VIC 3052, Australia

Dr Anna MH Price (corresponding author)

Murdoch Childrens Research Institute, and the Centre for Community Child Health, The Royal Children's Hospital.

50 Flemington Road, Parkville, VIC 3052, Australia

$+61399366613$

anna.price@mcri.edu.au

This is the author manuscript accepted for publication and has undergone full peer review but has not been through the copyediting, typesetting, pagination and proofreading process, which may lead to differences between this version and the Version of Record. Please cite this article as doi: $10.1111 /$ jpc.13860

This article is protected by copyright. All rights reserved. 


\begin{abstract}
Aims: Many, varied, antenatal risk factors can adversely impact children's health, behavior and cognition. Understanding the antenatal risk factors experienced by women can help identify which families are most likely to benefit from additional early supports; however, there is a dearth of published Australian antenatal risk data. This study aimed to determine the prevalence and co-occurrence of a broad range of risk factors in a community-based sample of pregnant Australian women.
\end{abstract}

Methods: Brief risk factor survey (10 items including age, social support, health, smoking, stress or anxious mood, education, household income, employment) collected from pregnant women attending the antenatal clinic waiting rooms of ten public maternity hospitals selected for their provision of care to areas with fewer socioeconomic resources in the states of Victoria and Tasmania, between May 2013-August 2014.

Results: 5586 women participated, with an average age of 29.0 years and gestation of 26.9 weeks. The most prevalent antenatal risk factors were poorer global health $(38.9 \%)$, not finishing high school (33.5\%) and smoking (14.9\%); all proportions higher than the general population. Most women reported at least 1 risk factor $(68.6 \%)$, with $21.5 \%$ reporting e 3 . There was a high degree of co-occurrence between risk factors, especially the three most prevalent.

Conclusions: This study reveals the considerable and varied risk burden experienced by Australian women during pregnancy. By understanding where need is greatest, and tailoring support accordingly, risk factor assessment provides an opportunity to address equity through health care, ultimately optimizing the future developmental outcomes of all children.

Key Words: Antenatal; Risk Factors; Socioeconomic Factors; Child Development; Learning 


\section{What is already known on this topic}

- A broad range of health, psychosocial and socioeconomic antenatal risk factors can adversely impact children's health, behavior and cognition.

- Understanding the antenatal risk factors experienced by women can help identify which families are most likely to benefit from additional early supports.

- Existing studies have examined the prevalence of individual or a limited number of antenatal risk factors, but few have examined the co-occurrence of a broad range of known adverse health, psychosocial and socioeconomic risks, and no Australian studies have surveyed these at a population level.

\section{What this paper adds}

- Using a brief, 10-item risk factor survey collected from $>5000$ Australian women, this study confirms the common, complex and diverse range of risk factors experienced by pregnant women attending antenatal care in Australia.

- There was a high co-occurrence between all items, especially the most prevalent. Women living in more disadvantaged neighborhoods reported more risk factors.

- Risk identification and assessment within Australia's excellent universal antenatal care platform would provide the opportunity to make better use of existing resources, and address equity through health care. 


\section{INTRODUCTION}

The links between women's antenatal risk factors and children's developmental trajectories are well-established, with risks broadly categorized into those related to the intrauterine environment (e.g. exposure to smoking, drugs and alcohol; maternal stress) and the psychosocial and socioeconomic context of a mother's life (e.g. maternal age, education, unemployment, health). ${ }^{1-8}$ Antenatal risk factors are not mutually exclusive and have been shown to impact on multiple domains of infant and child development including health, behavior and cognition. ${ }^{1-3}$

Importantly, there is a clear social gradient in the distribution of these risk factors. Families experiencing the greatest social adversity report a higher number of, and more severe, risk factors, and their children have the poorest outcomes. ${ }^{1,4}$ Given that many risk factors associated with adversity are potentially modifiable, it may be possible to improve outcomes for both women and children, and reduce or prevent the social gradient, by identifying women at risk during pregnancy and providing them with sufficient and effective supports and resources.

Existing studies have examined the prevalence of individual or a limited number of antenatal risk factors, ${ }^{4,7,9-15}$ but few have examined the co-occurrence of a broad range of known adverse health, psychosocial and socioeconomic risks, and no Australian studies have surveyed these at a population level. The closest comparable data available are from the Growing Up in New Zealand (NZ) longitudinal Study ( $n=6000),{ }^{5}$ which described the prevalence and co-occurrence of 12 antenatal risk factors such as teenage pregnancy, single parenthood, not finishing high school, smoking and financial stress. The study found a high prevalence of risk factors - ranging from $5 \%$ for teenage pregnancy to $28 \%$ living in areas in the lowest socioeconomic quintile (greatest disadvantage) - and a high level of cooccurrence. The morbidity load was considerable: two-thirds of women reported at least 1 risk factor and one-fifth reported 3 or more. ${ }^{5}$

The high prevalence of adverse risk factors reported in previous research ${ }^{4,7,9-15}$ suggests that antenatal health care platforms in Australia are well-placed to identify and address the factors that are most amenable to influence. However, the focus in Australian maternity hospitals or units tends to be on intrauterine risk factors, with far more variability in the approach to understanding and addressing the psychosocial and socioeconomic needs of women. ${ }^{16}$ In the absence of these data, it is difficult to determine how best to influence 
potential policy and practice changes, or even if the prevalence of risk factors justifies any changes to current approaches.

In an earlier pilot study ( $\mathrm{n}=186)$, we demonstrated that a brief, study-designed, 10item self-report survey assessing a broad range of health, psychosocial and socioeconomic risk factors is acceptable to pregnant women and feasible to collect in the waiting rooms of public antenatal clinics. ${ }^{17}$ This brief risk factor survey (hereafter referred to as BRF survey) provided a method for assessing risk factor data at the population level. It was subsequently collected from a large, prospective, population-based cohort of pregnant Australian women in the context of recruiting for a randomized controlled trial (RCT) of sustained nurse home visiting (SNHV, ISRCTN89962120). Using the BRF survey data, we aimed to investigate the prevalence; frequency and co-occurrence between risk factors. We hypothesized that (1) the prevalence of adverse antenatal risk factors would be high; (2) a higher count of risk factors would be associated with greater area level disadvantage; and (3) there would be a high degree of co-occurrence between risk factors, with the variability in observed risk being substantively explained by one or more common underlying factors.

\section{METHODS}

Survey design, participants and setting: This study-designed, cross-sectional BRF survey (see Measures, below) was nested within the "right@home” RCT. The RCT aims to test whether a SNHV program can improve the learning and development of children by school-entry, when delivered to families via the universal child and family health service which is available to all Australian families. ${ }^{18}$ The BRF survey was used to identify pregnant women who were eligible to participate in the RCT.

Most pregnant Australian women obtain free or heavily subsidized antenatal clinical care at large, public, maternity hospitals. ${ }^{19}$ Participants were pregnant women attending antenatal clinics at ten public maternity hospitals between May 2013 and August 2014. The ten hospitals were in metropolitan and regional areas of Victoria and Tasmania and selected for factors including their known provision of care to women living in areas with fewer socioeconomic resources and high birth rates (relative to other hospitals). Women were excluded if they had insufficient comprehension to consent (e.g. due to non-English speaking background (NESB) or intellectual disability). When clinics were too busy to survey all the women, they were screened for RCT exclusion criteria before completing the survey; these 
additional exclusion criteria included $>36$ weeks gestation, an expected date of delivery after 30 September 2014, or living outside the councils/regions involved in the RCT.

Procedure: Trained interviewers approached all women in the waiting rooms of the antenatal clinics and invited them to complete the BRF survey. Women were missed if they left the waiting rooms (e.g. to start or leave their appointment) before completing the survey. Women usually completed the survey alone but to accommodate low literacy, researchers offered to go through the survey with each woman if they preferred. Researchers checked all BRF surveys for completeness before manually entering the data into a customized, password-protected, online database.

Measures: We designed the cross-sectional BRF survey based on the existing antenatal and home visiting literature demonstrating associations between women's experience of adversity during pregnancy and children's later developmental outcomes. ${ }^{4,20,21}$ The self-report survey asked about a broad range of 10 known adverse risk factors (Table 1) in a way that was feasible for collection in public waiting rooms and acceptable to women. While the survey design attempted to capture the most important antenatal risks predictive of poorer child development outcomes, some were not included in the survey (e.g. antenatal substance use, family violence), which are profound risk factors for poorer child development but were too sensitive to ask in the waiting rooms. ${ }^{29,30}$ However, our earlier pilot study examined the associations between the BRF survey and the more sensitive antenatal risk data collected by midwives in the standard clinical appointments. The BRF survey identified counts of 1 adverse risk factor for $28 \%$ of the women surveyed in the pilot, 2 for $17 \%$, and 3 or more for $22 \%$. By linking these data, we demonstrated that using a classification of 1 or more, 2 or more or 3 or more risk factors identified $92 \%, 61 \%$ and $44 \%$, respectively, of women who reported other more sensitive risk factors (e.g. alcohol and drug use, domestic violence) in the standard clinical appointment setting. ${ }^{17}$ The BRF also recorded women's postcodes, which were linked with the Socio-Economic Indexes for Areas (SEIFA) Index of Relative Disadvantage, and Australian Standard Geographical Classification for Remoteness Area (ASGC-RA) (Table 1).

Samples size and analyses: The sample size was unrestricted with surveys collected until the necessary sample size for the RCT $(\mathrm{N}=714)$ was reached and recruitment ended. Participant characteristics and the prevalence of BRF survey items were described by tabulating proportions (categorical data) and means and standard deviations (continuous 
data), and calculating a count of the adverse BRF survey items (possible range 0-10) (Aim 1). Associations between SEIFA quintiles and count of adverse BRF survey items was investigated using the chi-squared test (Aim 2). Analysis for Aim 3 involved calculating Pearson $\mathrm{Chi}^{2}$ correlations between each pair of risk factors and conducting an exploratory factor analysis including all of the 10 risk factors. Although there were some missing data (range $0-10 \%$ across all items), we did not conduct multiple imputation due to the lack of repeated measures to inform the imputation model and concern regarding non-random reasons for missing data. Data were analyzed using Stata 13.0 (StataCorp, College Station, TX).

Ethical approval: This study was approved by the Human Research Ethics Committees of: The Royal Children's Hospital (HREC 32296); Peninsula Health (HREC/13/PH/14); Ballarat Health Services (HREC/13/BHSSJOG/9); Southern Health (HREC 13084X); Northern Health (HREC P03/13) in Victoria, Australia; and The University of Tasmania (HREC H0013113), Tasmania, Australia.

\section{RESULTS}

Participant characteristics: Figure 1 shows the participant flow. Of 9511 pregnant women approached, 667 were ineligible because they had insufficient English to participate or comprehend the study invitation. A further 1867 were not invited to complete the survey because they were not eligible for the RCT. Of the remaining 6977 eligible women, 5586 (80.1\%) completed the BRF survey, 923 (13.2\%) declined and 468 (6.7\%) left the waiting room before completing it.

Participating women had an average age of 29.0 years and average gestation of 26.9 weeks (Table 2). As the gestation criterion was sometimes clarified after enrolment in the RCT, 670 women completed the survey with gestation greater than 36 weeks and were included in the analyses. The socioeconomic profile of the cohort reflected the recruitment from hospitals serving women experiencing higher social adversity, with $28.1 \%$ from SEIFA quintile 1 (greatest disadvantage) compared with 20\% nationally, and 78.3\% from SEIFA quintiles $1-3$, compared with $60 \%$ nationally. Most women lived in major cities (48.0\%) or inner regional (40.6\%) areas according to ASGC Remoteness Area classification.

Prevalence and count of brief risk factor survey items: The prevalence of adverse risk factors in the population was high (Table 2). The most prevalent risks were poorer global 
health (38.9\%) and low education (33.3\%), while the least prevalent were lack of support in pregnancy (3.8\%) and not living with another adult (6.2\%). Over half of the women $(57.5 \%)$ experienced 1 to 3 risk factors, while $11.0 \%$ reported 4 to 8 (Table 3 ).

Social gradient of brief risk factor survey item count (Table 4): Women living in quintile 1 (greatest disadvantage) had higher rates of 4 or more adverse risk factors $(17.6 \%)$ compared to those living in quintile 5 (least disadvantage) (5.3\%). Less than a quarter (23.8\%) of women in quintile 1 reported a BRF count of zero, compared to $43.3 \%$ in quintile 5. A substantial proportion of women living in the third SEIFA quintile (average socioeconomic status) experienced multiple adverse risk factors, with 37.0\% reporting 2 or more.

Co-occurrence of adverse risk factors (Table 5): There was a high co-occurrence between most adverse risk factors, although substantial variation in these frequencies. Poorer global health was reported by $71.0 \%$ of women with chronic illness, $63.4 \%$ of women experiencing significant stress and $60.5 \%$ not living with another adult. Low education was experienced by $67.0 \%$ of women who reported currently smoking, $63.7 \%$ with young pregnancy, $62.4 \%$ with no household income and $60.8 \%$ not living with another adult. Of the 347 women not living with another adult, $70.9 \%$ had no income.

Conducting an exploratory factor analysis including all of the adverse risk factors identified a single underlying factor which substantively explained the variability in the observed risk (Eigenvalue 1.37). Examination of this factor found positive contributions of all of the observed risks; most prominently: no-one in the household with a paid income (Factor loading 0.62$)$, not living with another adult (0.52), smoking (0.44) and not finishing high school (0.41). No other underlying factors substantively explained variability in the observed risk factors (Eigenvalues 0.35 or less).

\section{DISCUSSION}

This study confirms the common, complex and diverse range of health, psychosocial and socioeconomic risk factors experienced by pregnant women attending antenatal care in Australia. The most prevalent adverse risk factors were poorer global health, not finishing high school, and smoking. There was a high co-occurrence between all items, especially the most prevalent. $68.6 \%$ of women in the current study reported at least 1 and $21.5 \%$ reported e 3 adverse risk factors. The BRF survey item count was socioeconomically-distributed, with 
women living in more disadvantaged neighborhoods reporting a higher BRF survey count. Factor analysis identified a single underlying construct that represented a combination of all the adverse risks; most predominantly: having no-one in the household with a paid income, not living with another adult, smoking, and not finishing high school.

It may be that the high prevalence of poorer self-rated health is related to the pregnancy itself, given the association with physical ailments such as morning sickness, heart burn, constipation, fatigue and back pain. ${ }^{31}$ We did not attempt to differentiate the acute changes associated with pregnancy with pre-existing conditions because this would likely need in-depth questions which would have been beyond the scope of the BRF survey. The relatively high prevalence of risk factors such as low education and the co-occurrence with other health risks such as smoking, suggests that reducing these risks could have immediate and longer-term positive consequences for women and their children. The high rates of cooccurrence likely reflects inter-related causal pathways. Of note are associations between low maternal education and smoking, young maternal age and the absence of another adult living in the household. Studies like Growing Up in NZ, ${ }^{5}$ the UK Millennium Cohort Study ${ }^{4}$ and a Canadian study by Santos et $\mathrm{al}^{8}$ demonstrated similar co-occurrences between maternal education, smoking, maternal age $<20$ years and single relationship status.

The current study has several strengths as the first, large, prospective, populationbased survey of risk factors in pregnant Australian women. The high response rate supports the results of the initial pilot study, ${ }^{17}$ suggesting that the survey is acceptable to women and feasible for use in the health care settings including those servicing areas of greater social adversity. It also provides a simple means for reaching a cohort that is frequently underrepresented in population-based, health services research.

There are a number of limitations. The cross-sectional design limits the interpretation of results to associations only, without being able to comment on the possible direction of relationships between risk factors. The generalizability of findings may also be limited. Data were collected from antenatal hospitals, and from clinics operating in areas known for their greater social adversity. While $72 \%$ of Australian women give birth in public hospitals, these findings may not represent the risk profiles of those giving birth in private hospitals or living in areas experiencing less adversity. ${ }^{32}$ Finally, with no data to compare the $13.2 \%$ of women who declined participation with the responding participants, there may be unmeasured selection bias. Women with the most complex needs and lives are often those most likely to 
be missed in community-based research, or present very late or not at all to antenatal care, thus the number and severity of risk factors may be higher for non-participating women. As such, the findings may underestimate the true prevalence and co-occurrence of risk factors at the population-level.

The high prevalence of adverse antenatal risk factors suggests there is an opportunity and imperative to more purposefully collect this information. Ensuring that social supports are in place to help reduce the negative effects of risk factors seems an important aspect of high quality antenatal care. Stimulating the antenatal health care system to consider a more systematic and consistent response to this risk morbidity load could have a substantial impact on a woman's pregnancy and the future health and development of her child.

\section{CONCLUSION}

Adverse antenatal risk factors for child development are prevalent, socioeconomically distributed and co-occur in pregnant Australian women. Many are modifiable and therefore amenable to early intervention strategies and support. While Australia's excellent universal antenatal care platform already conducts a number of risk factor assessments, our findings suggest that assessing a broader range of risk factors in a consistent manner across Australian antenatal clinics could help clinicians better understand women's psychosocial context and risk for their children. By understanding where need is greatest, and tailoring support accordingly, risk factor assessment provides an opportunity to address equity through health care, ultimately optimizing the future developmental outcomes of all children.

\section{ACKNOWLEDGEMENTS}

The "right@home" sustained nurse home visiting trial is a research collaboration between the Australian Research Alliance for Children and Youth (ARACY); the Translational Research and Social Innovation (TReSI) Group at Western Sydney University; and the Centre for Community Child Health (CCCH), which is a department of The Royal Children's Hospital and a research group of Murdoch Children's Research Institute. We thank all families, the research assistants, and nurses and social care practitioners working on the right@home trial, the antenatal clinic staff at participating hospitals who helped facilitate the research, and the Expert Reference Group for their guidance in designing the trial. 


\section{Funding Statement}

This work is supported by the Victorian Department of Education and Training, the Tasmanian Department of Health and Human Services, the Ian Potter Foundation, Sabemo Trust, Sidney Myer fund, the Vincent Fairfax Family Foundation, and the National Health and Medical Research Council (NHMRC, 1079418). The MCRI administered the research grant for the study and provided infrastructural support to its staff but played no role in the conduct or analysis of the trial. Research at the MCRI is supported by the Victorian Government's Operational Infrastructure Support Program. SG was supported by an NHMRC Career Development Award (1082922). FM was supported by NHMRC Early Career and Career Development Fellowships $(1037449,1111160)$. The funding bodies had no role in relation to the design and conduct of the study; collection, management, analysis, and interpretation of the data; preparation, review, or approval of the manuscript; and decision to submit the manuscript for publication. The authors have no conflicts of interest to declare.

Clinical Trial Registration: International Standard Randomized Controlled Trial Number ISRCTN89962120 (for larger randomized controlled trial). 


\section{REFERENCES}

1. Centre for Community Child Health and Telethon Institute for Child Health Research. A Snapshot of Early Childhood Development in Australia - Australian Early Development Index (AEDI) National Report. Canberra, Australia: Australian Government Department of Education; 2012.

2. Edwards B, Baxter J, Smart D, Sanson A, Hayes A. Financial disadvantage and children's school readiness. Family Matters. 2009(83):23-31.

3. National Scientific Council on the Developing Child. Early exposure to toxic substances damages brain architecture: Working Paper No. 4. Cambridge, MA: Harvard University; 2006.

4. Sabates R, Dex S. Multiple risk factors in young children's development. London: Centre for Longitudinal Studies, Institute of Education; 2012.

5. Morton SMB, Atatoa Carr PE, Grant CC, et al. Growing Up in New Zealand: A longitudinal study of New Zealand children and their families - Vulnerability Report 1: Exploring the definition of vulnerability for children in their first 1000 Days. Auckland, New Zealand: The University of Auckland; 2014.

6. National Scientific Council on the Developing Child. Excessive stress disrupts the architecture of the developing brain:Working Paper No. 3. Cambridge, MA: Harvard University; 2005.

7. Kiernan KE, Mensah FK. Maternal indicators in pregnancy and children's infancy that signal future outcomes for children's development, behaviour and health: Evidence from the Millennium Cohort Study. York, UK: University of York; 2009.

8. Santos R, Brownell M, Ekuma O, Mayer T, Soodeen R. The Early Development Instrument (EDI) in Manitoba: Linking Socioeconomic Adversity and Biological Vulnerability at Birth to Children's Outcomes at Age 5. Winnipeg, MB: Manitoba Centre for Health Policy; 2012.

9. Malacova E, Li J, Blair E, Mattes E, de Klerk N, Stanley F. Neighbourhood socioeconomic status and maternal factors at birth as moderators of the association between birth characteristics and school attainment: a population study of children attending government schools in Western Australia. J. Epidemiol. Community Health. 2009;63(10):842-849. 
10. Santos IS, Matijasevich A, Barros AJD, Barros FC. Antenatal and postnatal maternal mood symptoms and psychiatric disorders in pre-school children from the 2004 Pelotas Birth Cohort. J. Affect. Disord. 2004;164:112-117.

11. Evans J, Melotti R, Heron J, et al. The timing of maternal depressive symptoms and child cognitive development: a longitudinal study. Journal of Child Psychology \& Psychiatry. 2012;53(6):632-640.

12. Yost NP, Bloom SL, McIntire DD, Leveno KJ. A prospective observational study of domestic violence during pregnancy. Obstet. Gynecol. 2005;106(1):61-65.

13. Janssen PA, Holt VL, Sugg NK, Emanuel I, Critchlow CM, Henderson AD. Intimate partner violence and adverse pregnancy outcomes: A population-based study. American Journal of Obstetrics and Gynecology. 2003;188(5):1341-1347.

14. Batstra L, Hadders-Algra M, Neeleman J. Effect of antenatal exposure to maternal smoking on behavioural problems and academic achievement in childhood: prospective evidence from a Dutch birth cohort. Early Human Development. . 2003;75(1):21-33.

15. Flach $\mathrm{C}$, Leese M, Heron J, et al. Antenatal domestic violence, maternal mental health and subsequent child behaviour: a cohort study. BJOG: An International Journal of Obstetrics \& Gynaecology. 2011;118(11):1383-1391.

16. Department of Health and Ageing. Australian Health Ministers' Advisory Council, Clinical Practice Guidelines: Antenatal Care-Module 1. Canberra, Australia: Australian Government Department of Health and Ageing; 2012. http://www.health.gov.au/antenatal. Accessed 9 September 2016. 2012.

17. Authors. The feasibility and acceptability of a population-level antenatal risk factor survey: Cross-sectional pilot study. J. Paediatr. Child Health. 2017;53(6)572-577.

18. Authors.right@home: A randomised controlled trial of sustained nurse home visiting from pregnancy to child age 2 years, versus usual care, to improve parent care, parent responsivity and the home learning environment at 2 years. BMJ Open. 2017;7(3)e013307.

19. Australian Institute of Health and Welfare. Australian hospital statistics 2011-12. Health services series no. 50. Canberra, Australia: Australian Institute of Health and Welfare; 2013. 
20. Chittleborough CR, Lawlor DA, Lynch JW. Prenatal prediction of poor maternal and offspring outcomes: Implications for selection into intensive parent support programs. Maternal \& Child Health Journal. 2012;16(4):909-920.

21. Kemp L, Harris E, McMahon C, et al. Child and family outcomes of a long-term nurse home visitation programme: A randomised controlled trial. Arch. Dis. Child. 2011;96(6):533-540.

22. Australian Bureau of Statistics. Australian Bureau of Statistics Census of Population and Housing: Socio-Economic Indexes for Areas (SEIFA), Australia: Datapaks; 2011; http://www.abs.gov.au/websitedbs/censushome.nsf/home/seifa2011. Accessed December 2015.

23. Australian Bureau of Statistics. Australian Statistical Geography Standard (ASGS): Volume 5 - Remoteness Structure; 2011 http://www.abs.gov.au/AUSSTATS/abs@.nsf/DetailsPage/1270.0.55.005July\%20201 1?OpenDocument. Accessed 2 June 2016.

24. Kiernan KE. Becoming a young parent: A longitudinal study of associated factors. $\mathrm{Br}$. J. Sociol. 1997;48(3):406-428.

25. Sanson AV, Hawkins MT, Misson S. The Development and Validation of Australian Indices of Child Development—Part II: Validity Support. Child Indicators Research. 2010;3(3):293-312.

26. Mensah F, Hobcroft J. Childhood deprivation, health and development: associations with adult health in the 1958 and 1970 British prospective birth cohort studies. Journal of Epidemiology and Community Health. 2008;62(7):599-606.

27. Office for National Statistics. Census 2001 Key Statistics 21: Long Term Illness. London: Office for National Statistics; 2001. http://data.london.gov.uk/dataset/census2001-key-statistics-21-long-term-illness. Accessed 18 December 2015.

28. Matthey S, Valenti B, Souter K, Ross-Hamid C. Research report: Comparison of four self-report measures and a generic mood question to screen for anxiety during pregnancy in English-speaking women. Journal of Affective Disorders. 2013;148:347351.

29. O'Leary CM. Fetal alcohol syndrome: diagnosis, epidemiology, and developmental outcomes. Journal Of Paediatrics And Child Health. 2004;40(1-2):2-7. 
30. Kiely M, Gantz M, El-Khorazaty M, El-Mohandes A. Sequential screening for psychosocial and behavioural risk during pregnancy in a population of urban African Americans. BJOG: An International Journal of Obstetrics \& Gynaecology. 2013;120(11):1395-1402.

31. Abbott J, Bowyer L, Finn M. Obstetrics \& gynaecology : an evidence-based guide, $2^{\text {nd }}$ Edition. Chatswood, N.S.W. : Churchill Livingstone; 2014.

32. Australian Institute of Health and Welfare. Australia's mothers and babies 2014-in brief. Canberra, Australia: Australian Institute of Health and Welfare; 2016. 
Table 1: Description of brief risk factor survey items

\section{Survey Items}

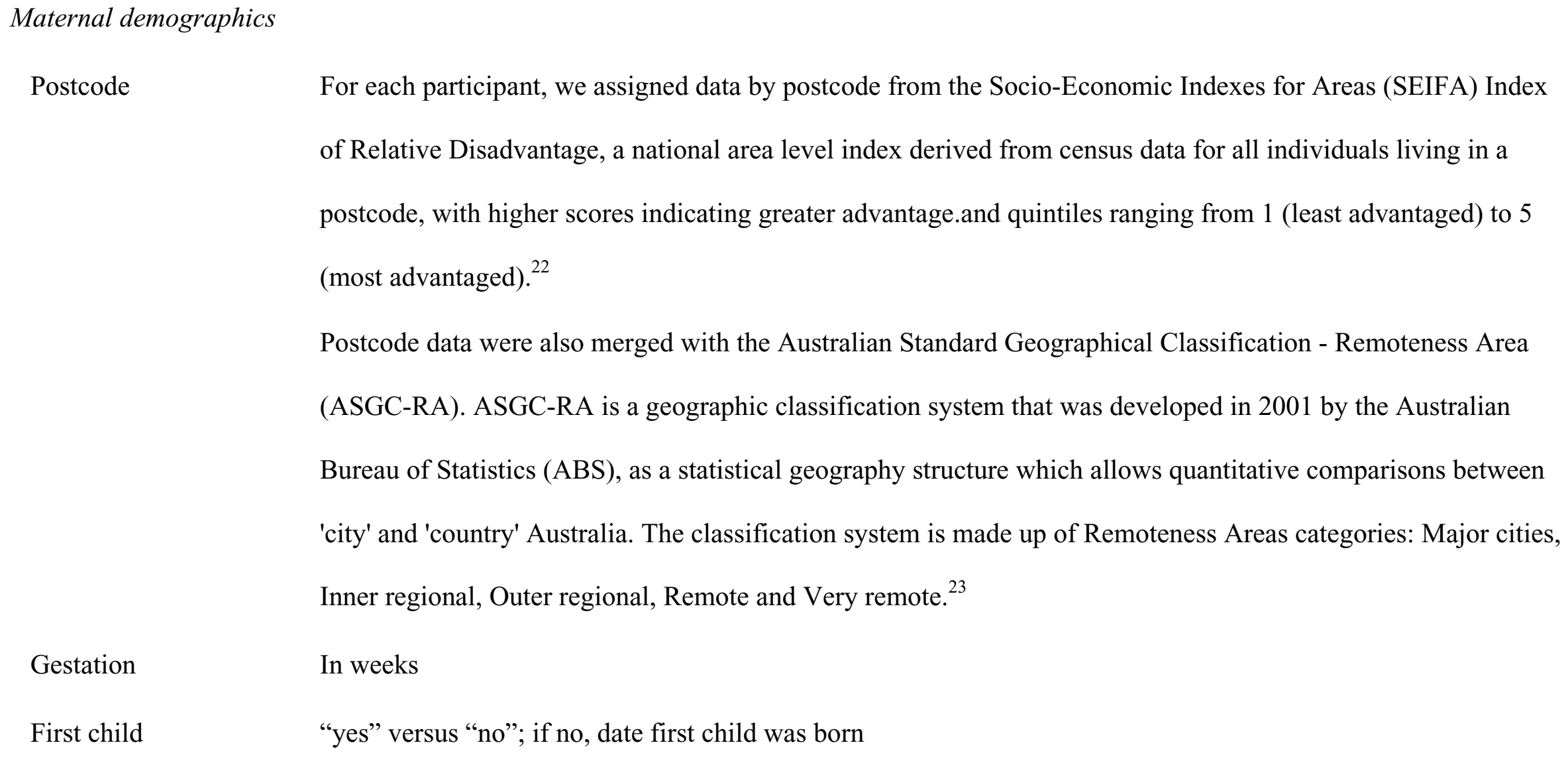

Postcode

For each participant, we assigned data by postcode from the Socio-Economic Indexes for Areas (SEIFA) Index of Relative Disadvantage, a national area level index derived from census data for all individuals living in a postcode, with higher scores indicating greater advantage.and quintiles ranging from 1 (least advantaged) to 5 (most advantaged). ${ }^{22}$

Postcode data were also merged with the Australian Standard Geographical Classification - Remoteness Area (ASGC-RA). ASGC-RA is a geographic classification system that was developed in 2001 by the Australian Bureau of Statistics (ABS), as a statistical geography structure which allows quantitative comparisons between 'city' and 'country' Australia. The classification system is made up of Remoteness Areas categories: Major cities, Inner regional, Outer regional, Remote and Very remote. ${ }^{23}$

Gestation In weeks

First child "yes" versus "no"; if no, date first child was born

Risk factors 
Table 1: Description of brief risk factor survey items

\section{Survey Items}

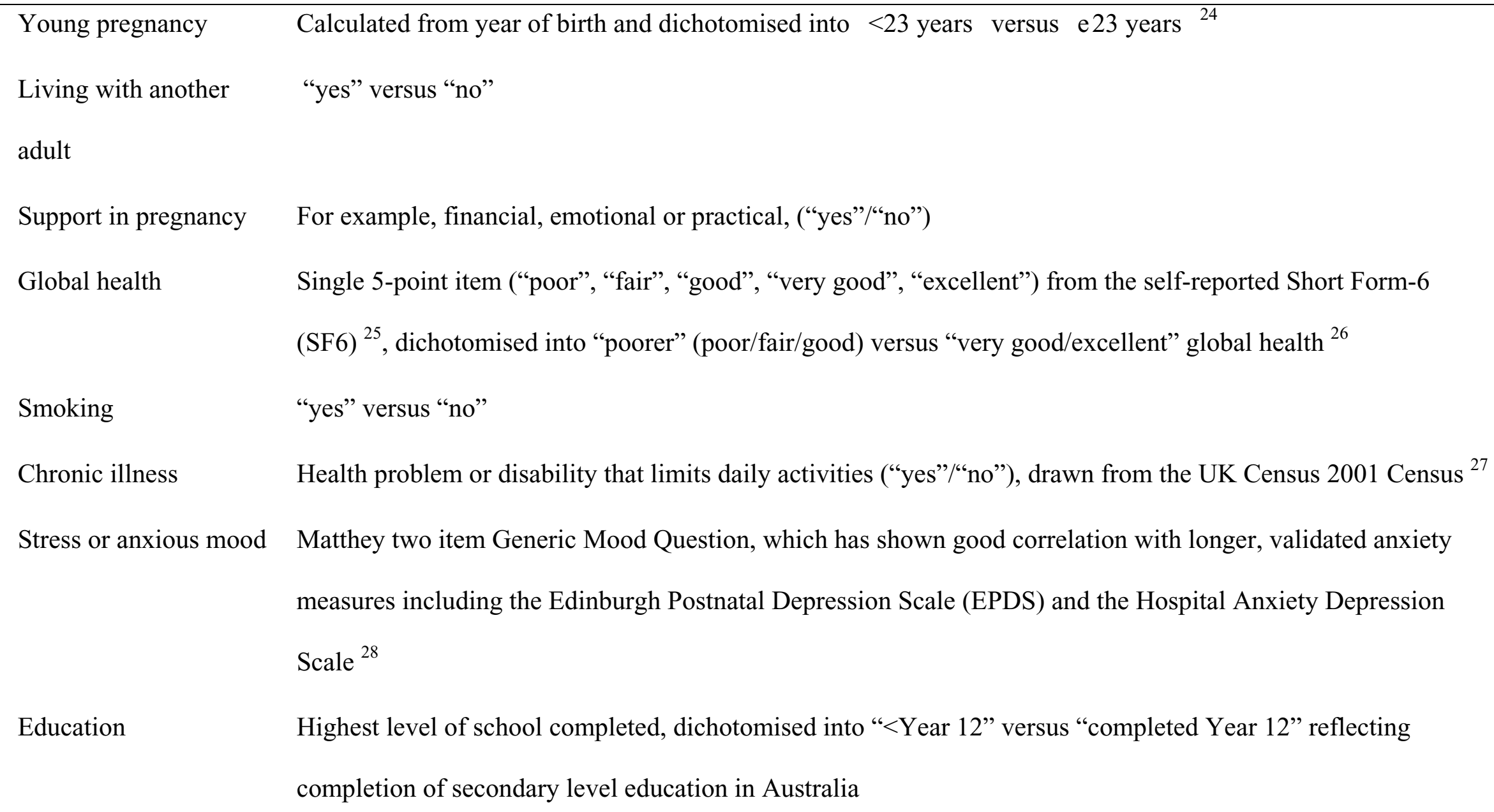

Chronic illness

Health problem or disability that limits daily activities (“yes"/“no"), drawn from the UK Census 2001 Census ${ }^{27}$

Stress or anxious mood Matthey two item Generic Mood Question, which has shown good correlation with longer, validated anxiety measures including the Edinburgh Postnatal Depression Scale (EPDS) and the Hospital Anxiety Depression Scale $^{28}$

Education Highest level of school completed, dichotomised into "<Year 12" versus "completed Year 12" reflecting completion of secondary level education in Australia 
Table 1: Description of brief risk factor survey items

Survey Items
Income
Person in the household currently has paid work/earns an income ("yes"/"no")
Ever worked
Participant has ever had a job before ("yes"/"no") 
Table 2: Participant demographics and prevalence of risk factors $\dagger$

Participant characteristics

n (\%) /

Mean (SD) [range]

\section{Demographics}

Maternal age in years, mean (SD), range

$29.0(5.6),[14-49]$

Gestation, weeks, mean (SD), range

$26.9(8.1),[3-43]$

Primiparous, $\mathrm{n}(\%)$

$2368(42.4)$

State, $\mathrm{n}(\%)$

Victoria

$4506(80.7)$

Tasmania

$1080(19.3)$

SEIFA Quintile, n (\%)

1 (greatest disadvantage)

$1563(28.1)$

2

$616(11.1)$

3

$2171(39.1)$

4

$890(16.0)$

5 (least disadvantage)

$319(5.7)$

ASGC Remoteness Area, n (\%)

Major Cities

$2668(48.0)$

Inner Regional

$2256(40.6)$

Outer Regional

$630(11.3)$

Remote

$6(0.1)$

Very Remote

$1(0.02)$

This article is protected by copyright. All rights reserved. 


\section{Participant characteristics}

n (\%) /

\section{Mean (SD) [range]}

Adverse risk factors according to the BRF survey, $\mathrm{n}(\%)$

Young maternal age $(<23$ years $)$

$735(13.2)$

Not living with another adult

$347(6.2)$

No support

Poorer global health

$2174(38.9)$

Smoking

Chronic illness

$451(8.1)$

Stress or anxious mood

Education $<$ Year 12

$1859(33.3)$

No household income

Never had a job

$385(6.9)$

† $\mathrm{N}$ ranged from 5511-5586 due to missing data

SEIFA: Socio-Economic Indexes for Areas (SEIFA) Index of Relative Disadvantage ASGC: Australian Standard Geographical Classification

BRF survey: Brief risk factor survey 
Table 3: Prevalence of brief risk factor survey count

\section{BRF survey Frequency Percentage}

\begin{tabular}{ccc} 
count & $(\mathbf{N})$ & $(\mathbf{\%})$ \\
\hline 0 & 1,753 & 31.4 \\
1 & 1,659 & 29.7 \\
2 & 975 & 17.5 \\
3 & 580 & 10.4 \\
4 & 320 & 5.7 \\
5 & 169 & 3.0 \\
6 & 97 & 1.7 \\
7 & 29 & 0.5 \\
8 & 4 & 0.1 \\
\hline TOTAL & 5,586 & \\
\hline BRF survey: Brief risk factor survey &
\end{tabular}

This article is protected by copyright. All rights reserved. 
Table 4: Brief risk factor survey count according to Socio-Economic Indexes for Areas Index of Relative Disadvantage

\begin{tabular}{ccccccc}
\hline SEIFA Quintile & $\mathbf{N}$ & \multicolumn{5}{c}{ BRF Survey Count (\%) } \\
\cline { 2 - 7 } & & $\mathbf{0}$ & $\mathbf{1}$ & $\mathbf{2}$ & $\mathbf{3}$ & $\mathbf{4 +}$ \\
\hline 1 (most disadvantaged) & 1563 & 23.8 & 25.3 & 20.3 & 13.1 & 17.6 \\
2 & 615 & 26.1 & 37.3 & 18.7 & 9.1 & 8.8 \\
3 & 2171 & 33.8 & 29.2 & 16.6 & 10.2 & 10.2 \\
4 & 890 & 38.2 & 33.4 & 14.6 & 8.3 & 5.5 \\
5 (least disadvantaged) & 319 & 43.3 & 29.2 & 15.1 & 7.2 & 5.3 \\
\hline
\end{tabular}

SEIFA: Socio-Economic Indexes for Areas Index of Relative Disadvantage BRF survey: Brief risk factor survey

$\mathrm{Chi}^{2}=223.28, \mathrm{p}<0.001$ 
Table 5: Co-occurrence of adverse risk factors in pregnancy

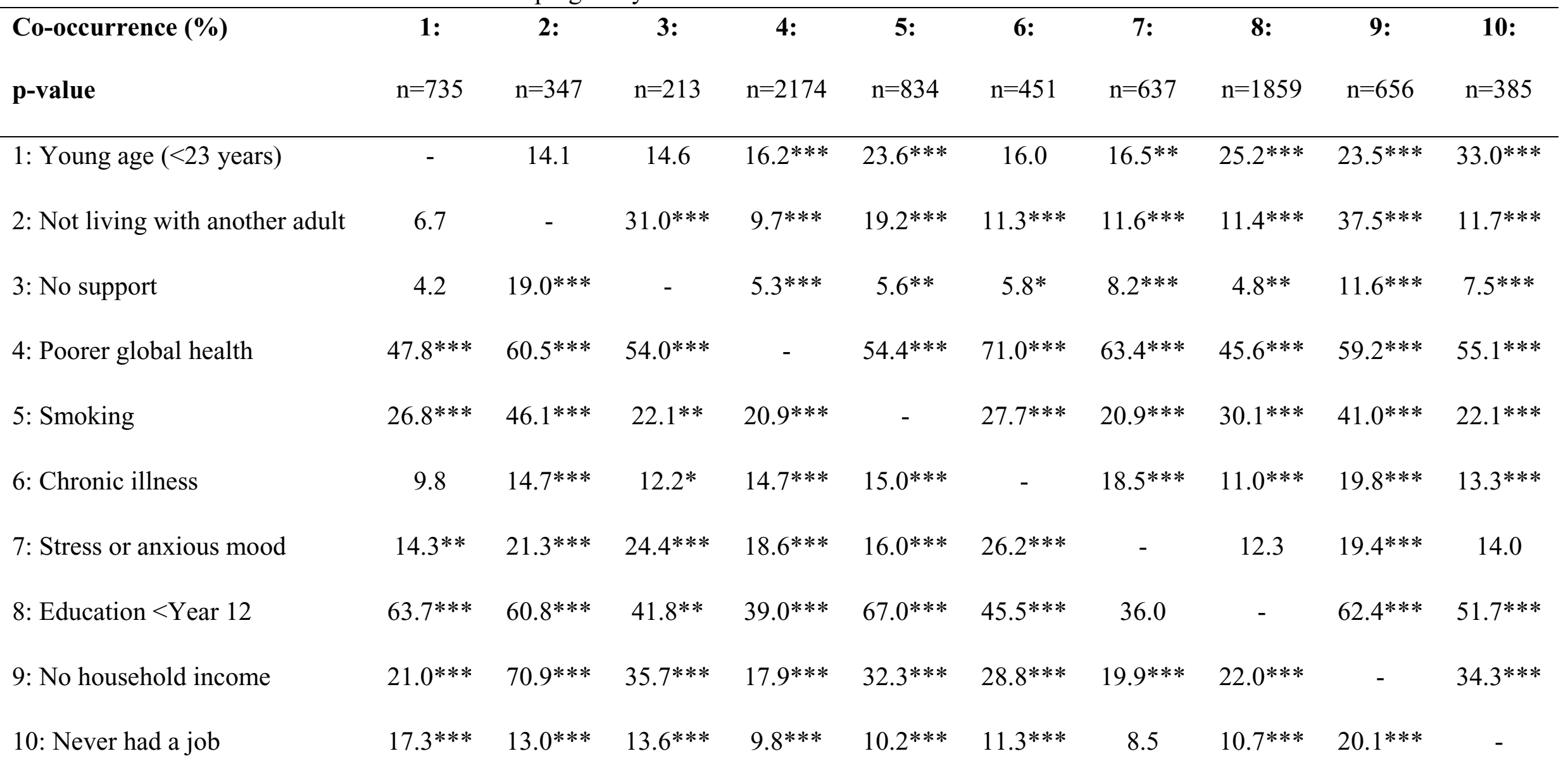

1: Young age (<23 years); 2: Not living with another adult; 3: No support during pregnancy; 4: Poorer global health (Poor/Fair/Good); 5: Current Smoking; 6: Long term illness or disability; 7: Stress or anxious mood; 8: Education < Year 12; 9: No household income; 10: Never had a job before. 
Please note: percentages are calculated by taking the risk factors listed at the LEFT as the numerator and the TOP as the denominator (e.g. Of 735 women $<23$ years old, 49 were not living with another adult (6.7\%), of 347 women not living with an adult, 49 were $<23$ years old $(14.1 \%)$ ). Significant Pearson $\mathrm{Chi}^{2}$ correlation between items at $* \mathrm{p}<0.05,{ }^{*} \mathrm{p}<0.01,{ }^{* * *}, \mathrm{p}<0.001$ 
Figure 1. Participant flow of brief risk factor (BRF) survey

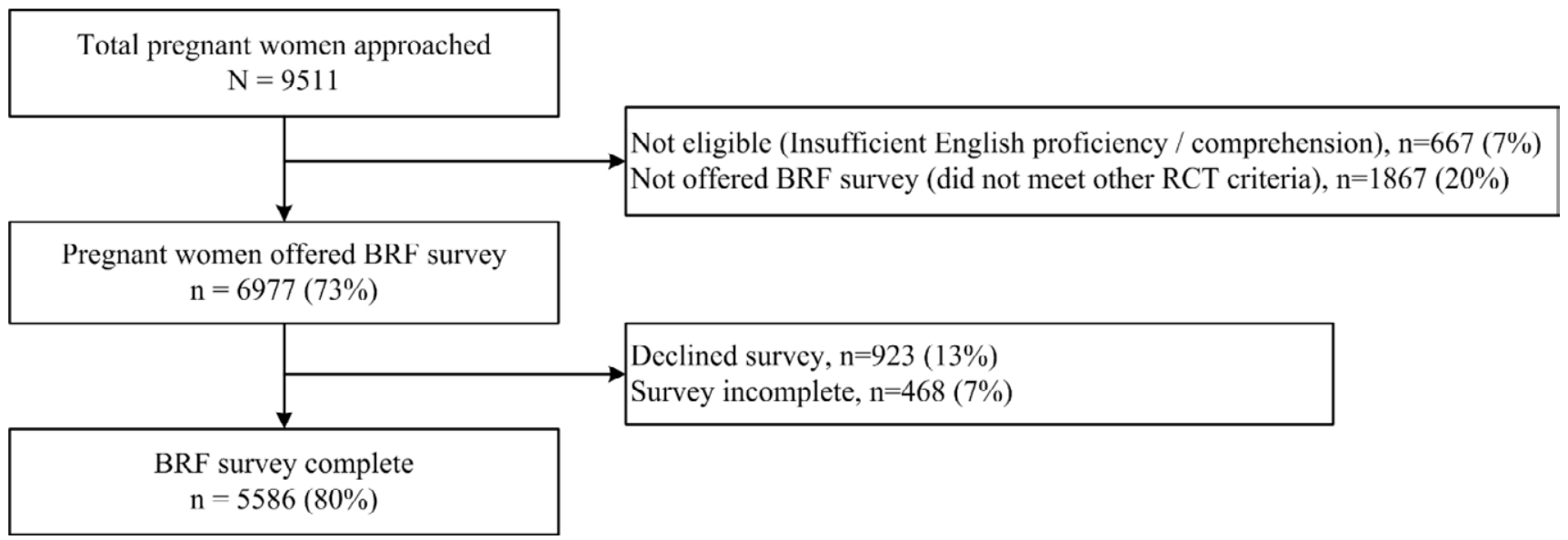


Figure 1. Participant flow of brief risk factor (BRF) survey

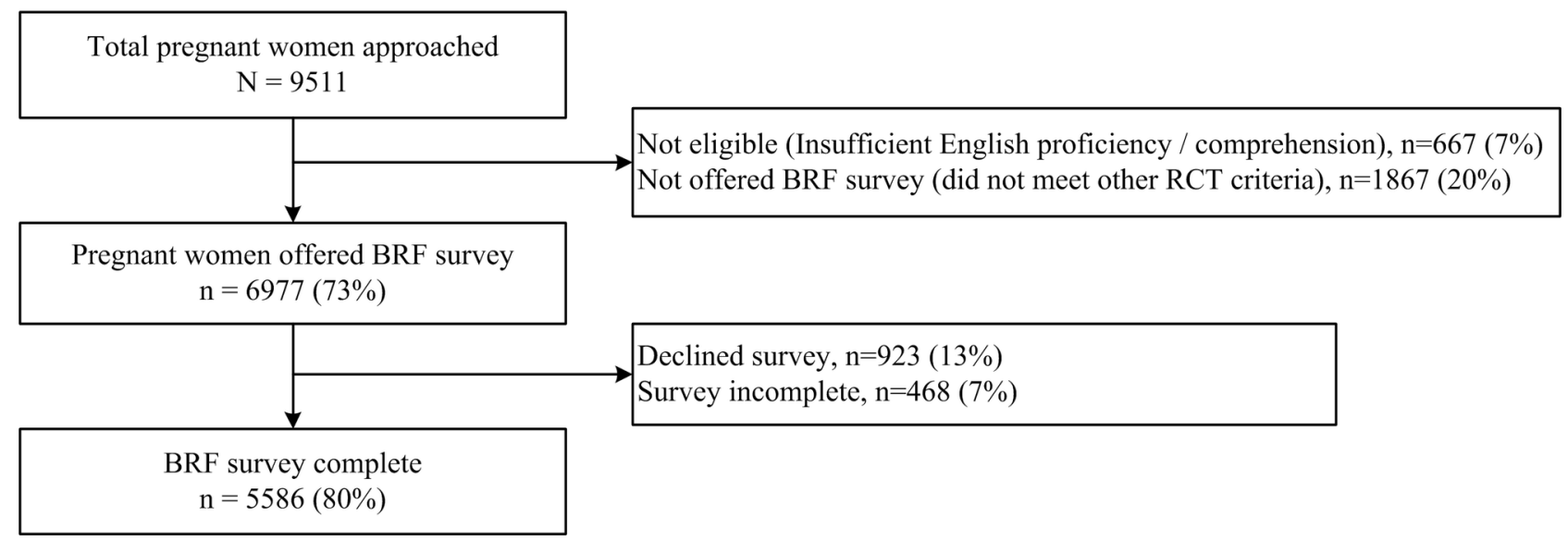

JPC_13860_Figure 1.tif 


\section{University Library}

\section{- $\mathrm{M}$ IIN E R VA A gateway to Melbourne's research publications}

Minerva Access is the Institutional Repository of The University of Melbourne

Author/s:

Goldfeld, S;D'Abaco, E;Bryson, H;Mensah, F;Price, AMH

Title:

Surveying social adversity in pregnancy: The antenatal risk burden experienced by Australian women

Date:

2018-07-01

Citation:

Goldfeld, S., D'Abaco, E., Bryson, H., Mensah, F. \& Price, A. M. H. (2018). Surveying social adversity in pregnancy: The antenatal risk burden experienced by Australian women. JOURNAL OF PAEDIATRICS AND CHILD HEALTH, 54 (7), pp.754-760. https:// doi.org/10.1111/jpc.13860.

Persistent Link:

http://hdl.handle.net/11343/283624 